\title{
Feasibility of imaging in nuclear magnetic resonance force microscopy using Boltzmann polarization
}

Cite as: J. Appl. Phys. 125, 083901 (2019); https://doi.org/10.1063/1.5064449

Submitted: 04 October 2018. Accepted: 03 February 2019. Published Online: 26 February 2019

M. de Wit (D), G. Welker (D), J. J. T. Wagenaar, F. G. Hoekstra (iD, and T. H. Oosterkamp (iD)

Oponocess

\section{ARTICLES YOU MAY BE INTERESTED IN}

Controlling the p-type conductivity of SnO by doping with nitrogen and hydrogen

Journal of Applied Physics 125, 085703 (2019); https://doi.org/10.1063/1.5052606

Impedance spectroscopy of Al/AIN/n-Si metal-insulator-semiconductor (MIS) structures

Journal of Applied Physics 125, 084501 (2019); https://doi.org/10.1063/1.5050181

The role of laser fluence and ambient environments on femtosecond laser induced breakdown spectroscopy and on surface morphology of $\mathrm{Mg}$ and $\mathrm{Zr}$

Journal of Applied Physics 125, 083302 (2019); https://doi.org/10.1063/1.5063897

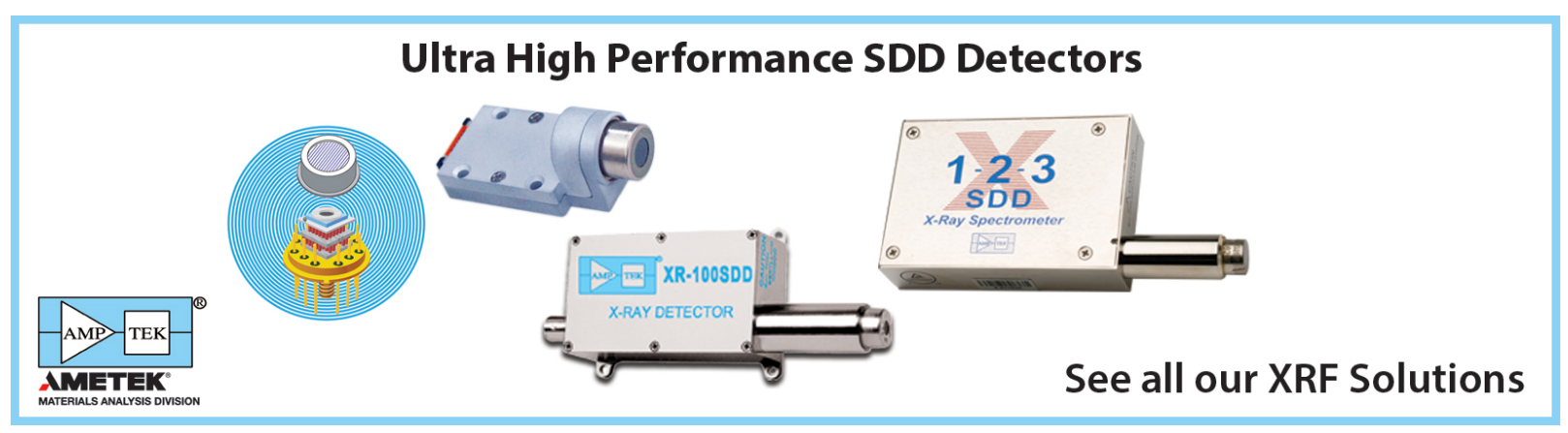




\title{
Feasibility of imaging in nuclear magnetic resonance force microscopy using Boltzmann polarization
}

Cite as: J. Appl. Phys. 125, 083901 (2019); doi: 10.1063/1.5064449

Submitted: 4 October 2018 - Accepted: 3 February 2019 .

Published Online: 26 February 2019

M. de Wit, (D) G. Welker, (D) J. J. T. Wagenaar, F. G. Hoekstra, (D) and T. H. Oosterkamp ${ }^{\text {) (D) }}$

\author{
AFFILIATIONS \\ Leiden Institute of Physics, Leiden University, PO Box 9504, 2300 RA Leiden, The Netherlands
}

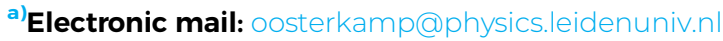

\begin{abstract}
We report on magnetic resonance force microscopy measurements of the Boltzmann polarization of nuclear spins in copper by detecting the frequency shift of a soft cantilever. We use the time-dependent solution of the Bloch equations to derive a concise equation describing the effect of radio-frequent (RF) magnetic fields on both on- and off-resonant spins in high magnetic field gradients. We then apply this theory to saturation experiments performed on a $100 \mathrm{~nm}$ thick layer of copper, where we use the higher modes of the cantilever as a source of the RF field. We demonstrate a detection volume sensitivity of only $(40 \mathrm{~nm})^{3}$, corresponding to about $1.6 \times 10^{4}$ polarized copper nuclear spins. We propose an experiment on protons where, with the appropriate technical improvements, frequency-shift based magnetic resonance imaging with a resolution better than $(10 \mathrm{~nm})^{3}$ could be possible. Achieving this resolution would make imaging based on the Boltzmann polarization competitive with the more traditional stochastic spin-fluctuation based imaging, with the possibility to work at millikelvin temperatures.
\end{abstract}

(C) 2019 Author(s). All article content, except where otherwise noted, is licensed under a Creative Commons Attribution (CC BY) license (http://creativecommons.org/licenses/by/4.0/). https://doi.org/10.1063/1.5064449

\section{INTRODUCTION}

Magnetic resonance force microscopy (MRFM) is a technique that combines magnetic resonance protocols with an ultrasensitive cantilever to measure the forces exerted by extremely small numbers of spins, with the immense potential of imaging biological samples with nanometer resolution. ${ }^{1-3}$ In the last 20 years, great steps have been taken towards this goal, with some milestones including the detection of a single electron spin, ${ }^{4}$ the magnetic resonance imaging of a tobacco mosaic virus with a spatial resolution of $4 \mathrm{~nm},{ }^{5}$ and more recently the demonstration of a one-dimensional slice thickness below $2 \mathrm{~nm}$ for the imaging of a polystyrene film. ${ }^{6}$ The experiments are typically performed by modulating the sample magnetization in resonance with the cantilever and then measuring either the resulting change in the oscillation amplitude (force-based) or the frequency shift (forcegradient based).

Both the force-based and force-gradient based experiments have some severe technical drawbacks, mainly associated to the cyclic inversion of the spin ensemble. For the coherent manipulation of the magnetization, alternating magnetic fields on the order of several $\mathrm{mT}$ are required., The dissipation associated with the generation of these fields is significant and prevents experiments from being performed at millikelvin temperatures, even for low duty-cycle MRMF protocols like cyclic-CERMIT. ${ }^{9,10}$ Furthermore, the requirement that the magnetization is inverted continuously during the detection of the signal means only samples with a long rotating-frame spin-lattice relaxation time $\mathrm{T}_{1 \rho}$ are suitable.

For imaging of nuclei, previous experiments have almost exclusively focused on measuring the statistical polarization of the spin ensemble. However, the possibility to use the Boltzmann polarization instead would dramatically improve the efficiency of the measurement, as averaging $\mathrm{N}$ times enhances the power signal-to-noise ratio (SNR) by a factor of $\mathrm{N}$ for Boltzmann based measurements, compared to $\sqrt{\mathrm{N}}$ for statistical polarization signals. There have been MRFM experiments based on the Boltzmann polarization, for instance, in 
order to measure the relaxation times of nuclei, ${ }^{9,11,12}$ but these experiments lacked the volume sensitivity required for imaging with a spatial resolution comparable to the statistical experiments.

In this work, we present measurements of the Boltzmann polarization of a copper sample at a temperature of $21 \mathrm{mK}$ by detecting the frequency shift induced by a saturation experiment. We derive the time-dependent solution to the Bloch equations appropriate for typical MRFM experiments, obtaining a concise equation for the non-equilibrium response of both on- and off-resonant spins to a radio-frequent (RF) pulse. Furthermore, we demonstrate that we can use higher modes of the cantilever as the source of the alternating field in order to generate the required RF fields to saturate the magnetization of the spins with minimal dissipation. ${ }^{13}$ These results suggest that imaging based on the Boltzmann polarization could be possible, allowing for the first MRFM imaging experiments performed at temperatures down to $10 \mathrm{mK}$ and using the magnet-on-tip geometry, as opposed to the sample-on-tip geometry more commonly found. We substantiate this claim by using the specifications of the current experiments to calculate the resolution for an imaging experiment on protons based on measuring the Boltzmann polarization.

\section{METHODS}

\section{A. Experimental setup}

We improve on earlier measurements in our group on nuclear spins in a copper sample. The setup and measurement procedure strongly resemble those used in that previous work. ${ }^{12}$ The operating principle of the MRFM is shown in Fig. 1(a). The heart of the setup is a soft single-crystal silicon cantilever (spring constant $k_{0}=70 \mu \mathrm{N} \mathrm{m}^{-1}$ ) 14 with a magnetic particle at the end with a radius $R_{0}=1.7 \mu \mathrm{m}$, resulting in a natural resonance frequency $f_{0}=\omega_{0} /(2 \pi) \sim 3.0 \mathrm{kHz}$, an intrinsic $\mathrm{Q}$-factor $\mathrm{Q}_{0} \sim 3 \times 10^{4}$, and a thermal force noise at $20 \mathrm{mK}$ of $0.4 \mathrm{aN} / \sqrt{\mathrm{Hz}}$. The magnet induces a static magnetic field $\mathrm{B}_{0}$ which can be well approximated by the field of a perfect magnetic dipole. The strength of the field of the magnet reduces quickly as the distance to the center of the magnet increases, creating a large magnetic field gradient. For typical experimental parameters, the magnetic field is of the order of a few tens to a few hundred $\mathrm{mT}$, with magnetic field gradients of approximately $100 \mathrm{mT} / \mu \mathrm{m}$. When the cantilever is placed at a height $h$ above a sample, spins in the sample couple to the resonator via the magnetic field gradient, inducing a frequency shift (see Sec. II E). An RF pulse with a frequency $\omega_{R F}$ can be used to remove the polarization of the spins that are resonant with this pulse, i.e., the spins that are within the resonant slice where $\left|B_{0}\right|=\omega_{R F} / \gamma$, with $\gamma$ the gyromagnetic ratio of the spins [in Fig. 1(a) the resonant slice is marked in red]. We will refer to this procedure as a saturation experiment or saturation pulse. The theoretical background of the saturation experiment is given in Sec. II D.

Our particular MRFM setup is designed to be operated at temperatures close to $10 \mathrm{mK}$ using a detection scheme based (a)
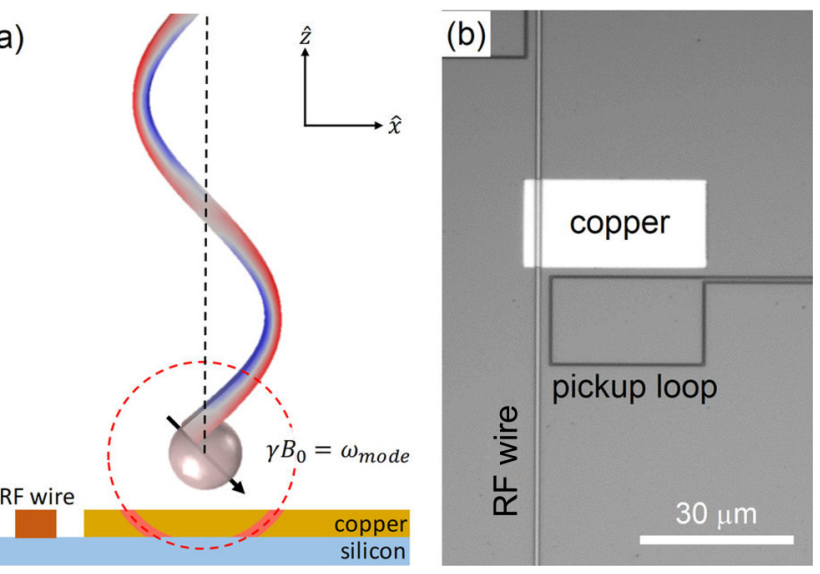

FIG. 1. (a) Schematic of the measurement setup. An RF wire is used to generate an RF field $B_{R F}$ directly or to excite higher modes of the cantilever to generate $B_{R F}$ fields with minimal dissipation. The RF pulse removes the Boltzmann polarization of spins located within and near the resonant slice (red region), inducing $\mathrm{mHz}$ shifts of the cantilever's fundamental resonance frequency. (b) Optical microscope image of the detection chip, showing the NbTiN pickup loop and RF wire, and the copper sample with a thickness of $100 \mathrm{~nm}$.

on a pickup loop [shown in Fig. 1(b)] and superconducting quantum interference device (SQUID). ${ }^{15}$ Additionally, we use a superconducting NbTiN RF wire to send RF currents to the sample. ${ }^{16}$ The MRFM setup is mounted at the bottom of a mechanical vibration isolation stage, and the cryostat has been modified to reduce vibrations originating from the pulse tube refrigerator. ${ }^{17,36}$

The RF pulse can be applied using two methods, both shown in Fig. 1(a). First of all, we can use an RF wire to send an alternating current, which generates a magnetic field directly. This allows for the precise control of the pulse shape and amplitude but at the cost of some heating of the sample due to $\mathrm{AC}$ dissipation in the superconducting $\mathrm{RF}$ wire. The amplitude of the RF field is given by $B_{R F}=\mu_{0} I / 2 \pi r$, with $r$ the distance to the RF wire, dictating that all measurements have to be done as close to the RF wire as possible (preferably within several micrometers). At a distance of $5 \mu \mathrm{m}$ from the RF wire, we can generate magnetic fields (in the rotating frame of the spins, see Sec. II D) of up to $0.3 \mathrm{mT}$. An alternative method to generate the required RF field is by using the higher modes of the cantilever, the proof of concept of which was recently demonstrated by Wagenaar et al. ${ }^{13}$ Generating RF fields using the higher modes can be done with a much smaller current in the RF wire to generate a magnetic drive field or by using a piezo at the base of the cantilever, allowing experiments at larger distances from the RF wire or even without one. In our experiment, we use a small current in the $\mathrm{RF}$ wire (on the order of $\sim 10 \mu \mathrm{A}$ ) to excite one of the higher modes of the cantilever, as illustrated in Fig. 1(a). The motion of the higher mode induces a small rotation of the magnet, which results in the generation of an amplified $B_{R F}$ at the frequency of the excited higher mode perpendicular to the tip 
field. In this way, RF fields can be generated with negligible dissipation.

The copper sample used in the experiment is patterned on the detection chip close to both the RF wire and the pickup loop, as shown in Fig. 1(b). The copper sample is a sputtered film with a thickness of $100 \mathrm{~nm}$, capped with a $20 \mathrm{~nm}$ layer of gold to prevent oxidation. The thickness of the sample was chosen to be $100 \mathrm{~nm}$ in order to reduce eddy currents in copper, which deteriorate the Q-factor of the cantilever and thereby the measurement sensitivity (for metal films with a thickness less than the skin depth, eddy current dissipation scales with the cube of the thickness ${ }^{18}$ ). Copper overlaps with the RF wire in order to give the sample a well defined potential. Besides the thermal conductance of the silicon substrate, there is no additional thermalization used to cool copper. The cantilever can be positioned above copper with a lateral accuracy of several micrometers. The relevant nuclear magnetic resonance (NMR) properties of copper for an MRFM experiment are detailed in the supplementary material.

\section{B. Frequency noise}

We have employed a series of improvements to the setup to enhance the frequency noise floor of the measurement and thus increase the sensitivity. The improvement is obvious when looking at the noise spectrum of the frequency, as shown in Fig. 2. The spectrum is measured by driving the cantilever with an amplitude of $43 \mathrm{~nm}_{\mathrm{rms}}$ and tracking the resonance frequency using a phase-locked loop (PLL) of a Zurich Instruments lock-in amplifier with a detection bandwidth of $40 \mathrm{~Hz}$. The PLL feedback signal is sent to a spectrum analyzer. In black we see the frequency noise spectrum of the current setup, while in red we see the frequency noise spectrum from

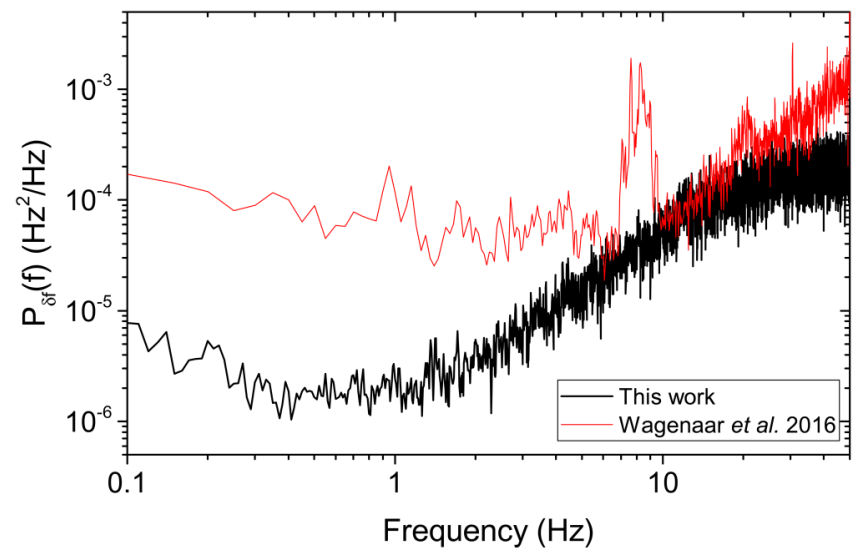

FIG. 2. Frequency noise spectrum $P_{\delta f}$ measured at a height of $1.3 \mu \mathrm{m}$. In red, we see the frequency noise spectrum from the initial experiment in our group, measured with a cantilever amplitude of $110 \mathrm{~nm}_{\mathrm{rms}}{ }^{12}$ In black, we see the current experiment, measured with a cantilever amplitude of $43 \mathrm{~nm}_{\mathrm{rms}}$. The reduction of the noise at higher frequencies in the black spectrum is due to the bandwidth of the PLL, which was set at $40 \mathrm{~Hz}$. the experiment in 2016 on a $300 \mathrm{~nm}$ thick copper film performed in our group. ${ }^{12}$ Both spectra were measured at a height of $1.3 \mu \mathrm{m}$ above a copper sample. The total frequency noise is given by the sum of the thermal noise, the detection noise, and the $1 / f$ noise typically attributed to the sample $e^{12,19}$

$$
P_{\delta f}(f)=P_{\delta f}^{\text {thermal }}+P_{\delta f}^{\text {det }} f^{2}+P_{\delta f}^{\text {sample }} f^{-1}
$$

The noise reduction of nearly 2 orders of magnitude is due to a combination of several technical improvements. Improved vibration isolation and cantilever thermalization have reduced the thermodynamic temperature of the cantilever from 132 $\mathrm{mK}$ to less than $50 \mathrm{mK}$. An improved design of the pickup loop resulted in an amplitude detection noise floor of $30 \mathrm{pm} / \sqrt{\mathrm{Hz}}$, determined from the measured transfer between the cantilever motion and the SQUID's output voltage. This allows for a much lower cantilever drive amplitude with the same detection frequency noise. The biggest improvement seems to be the reduction of the thickness of the copper film. Because the dissipated power of the eddy currents in the film scales strongly with the thickness of the film, we find that the measured Q-factor at $1.3 \mu \mathrm{m}$ from the sample has increased from 317 for the $300 \mathrm{~nm}$ film to almost 5000 for the $100 \mathrm{~nm}$ film. This reduces all three contributions to the frequency noise, particularly the $1 / f$ noise which is mainly attributed to eddy currents in the sample. The thermal noise floor using these parameters is estimated to be $0.7 \mathrm{mHz} / \sqrt{\mathrm{Hz}}$, so the data in Fig. 2 are not thermally limited. With a $1 \mathrm{~Hz}$ detection bandwidth, the integrated frequency noise is as low as $1.8 \mathrm{mHz}$.

\section{Measurement procedure}

A typical saturation recovery measurement (performed at a temperature $\mathrm{T}=40 \mathrm{mK}$ ) is shown in Fig. 3. Again a PLL is used to measure the frequency shift $\Delta f=f(t)-f_{0}$. At $t=0$, an RF pulse with a certain duration $t_{p}$ and strength $B_{R F}$ is turned on. The start and end are indicated by the green and orange vertical lines in Fig. 3. During the pulse, we observe frequency shifts that we attribute to a combination of electrostatic effects and slight local heating of the sample. After the pulse, the frequency shift relative to $f_{0}$ is measured. The obtained recovery curve can be fitted to

$$
\Delta f(t)=\Delta f_{0} e^{-\left(t-t_{0}\right) / T_{1}},
$$

with $\Delta f_{0}$ the direct frequency shift at time $t_{0}$, the end of the pulse. The light blue curve in Fig. 3 shows the result of a single measurement of the frequency shift (with a $1 \mathrm{~Hz}$ low-pass filter), and the dark blue curve shows the result of 50 averages. In red we show the best fit to the data using Eq. (2).

\section{Spin dynamics in MRFM}

In order to fully understand the observed frequency shifts, we need to find the final magnetization of the spins 


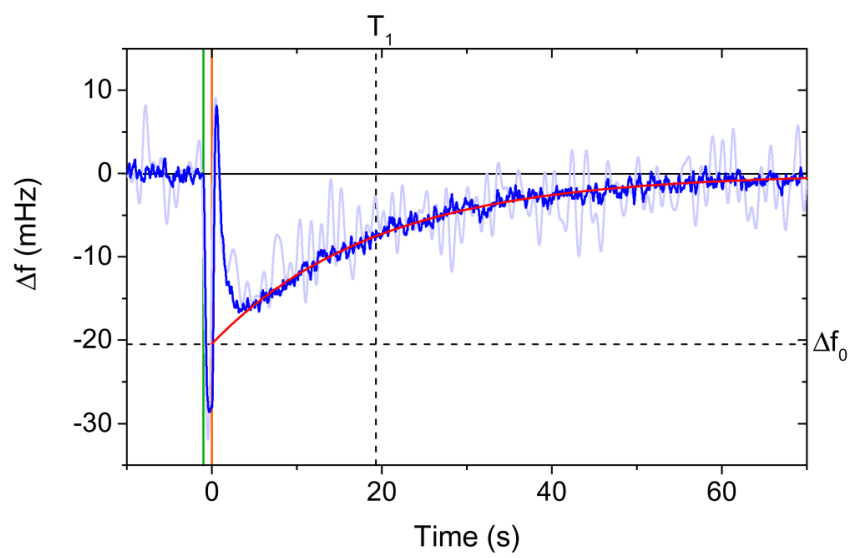

FIG. 3. Example of a typical measurement (at $T=40 \mathrm{mK}$ ) where we show the frequency shift $\Delta f$ with respect to the equilibrium frequency $f_{0}$. The light blue line shows a single measurement of the frequency shift (after a $1 \mathrm{~Hz}$ low-pass filter). The dark blue line shows 50 averages. The red solid line is an exponential fit to the data following Eq. (2). The green and orange vertical lines indicate the start and end of the saturation pulse.

coupled to the magnetic field of our cantilever after a saturation pulse. The behaviour of spins in alternating magnetic fields is well understood from conventional NMR, but the analysis is often limited to steady-state solutions. ${ }^{20}$ This limit works well for most NMR applications where the alternating fields are of sufficient strength and duration that the magnetization of the spin ensemble has reached an equilibrium during the pulse, but this does not necessarily work for MRFM due to the large magnetic field gradient, resulting large number of off-resonant spins, and the often weak oscillating magnetic fields. Therefore, we will derive equations for the time dependence of the magnetization of spins during an RF pulse, also for spins not meeting the resonance condition. These equations are then used to derive the effective resonant slice thickness in an MRFM experiment, a crucial component trying to decrease the detection volume and thereby optimize the imaging resolution.

The time evolution of spins subjected to a large static magnetic field $\left(B_{0}\right)$ and a small alternating magnetic field $\left(B_{R F}\right)$ perpendicular to the static field has long been understood using the Bloch equations. ${ }^{21}$ In the rotating frame, the equations of motion of the magnetization $\boldsymbol{m}(\mathrm{t})$ subjected to an effective magnetic field $\mathbf{B}_{\text {eff }}=\left(B_{0}-\omega / \gamma\right) \hat{\boldsymbol{k}}+B_{R F} \hat{\boldsymbol{i}}$ are given by

$$
\begin{aligned}
& \frac{d m_{x}}{d t}=-\Delta \omega m_{y}-\frac{m_{x}(t)}{T_{2}}, \\
& \frac{d m_{y}}{d t}=\omega_{1} m_{z}+\Delta \omega m_{x}-\frac{m_{y}(t)}{T_{2}}, \\
& \frac{d m_{z}}{d t}=-\omega_{1} m_{y}-\frac{m_{z}(t)-m_{0}}{T_{1}} .
\end{aligned}
$$

Here, $\gamma$ is the gyromagnetic ratio of the spins, $T_{1}$ and $T_{2}$ are the spin-lattice (longitudinal) and spin-spin (transverse) relaxation times, the detuning $\Delta \omega \equiv \omega-\omega_{0}$ with $\omega_{0}=\gamma B_{0}$ the Larmor frequency, and $\omega_{1} \equiv \gamma \mathrm{B}_{\mathrm{RF}} . m_{0}$ is the initial magnetization in thermal equilibrium. $\hat{\boldsymbol{k}}$ is the unit vector pointing in the direction of the $B_{0}$ field. To solve this system of differential equations, it is convenient to rewrite them in vector notation as

$$
\dot{m}=\mathrm{A} \boldsymbol{m}+\boldsymbol{b},
$$

with the source term $\boldsymbol{b}=\frac{m_{0}}{T_{1}} \hat{\boldsymbol{k}}$, and A given by

$$
A=\left(\begin{array}{ccc}
-\frac{1}{T_{2}} & -\Delta \omega & 0 \\
\Delta \omega & -\frac{1}{T_{2}} & \omega_{1} \\
0 & -\omega_{1} & -\frac{1}{T_{1}}
\end{array}\right)
$$

The steady state solution is now easy to derive by solving the differential equation after setting $\dot{\boldsymbol{m}}=0$. Note that $m_{x}$ and $m_{y}$ are rotating with the Larmor frequency around the z-axis. As the resonance frequencies of the cantilevers used in MRFM are typically much lower than the Larmor frequency, any coupling of these two components to the cantilever averages out over time. Therefore, we are only interested in the $\mathrm{z}$-component of the magnetization, which is the same in the rotating frame as in the laboratory frame 20,22

$$
\begin{aligned}
m_{z, \infty} & =\frac{1+\Delta \omega^{2} \mathrm{~T}_{2}^{2}}{1+\Delta \omega^{2} \mathrm{~T}_{2}^{2}+\omega_{1}^{2} \mathrm{~T}_{1} \mathrm{~T}_{2}} m_{0} \\
& \equiv p_{z} m_{0} .
\end{aligned}
$$

In the last line, we defined $p_{z}$ as the fraction of the magnetization that is removed by the $B_{R F}$ field if it is left on continuously.

In MRFM experiments, the steady state solution described by Eq. (6) is often not enough, as the RF pulses are not necessarily of sufficient strength and duration to fully saturate the magnetization of a spin ensemble. The timedependent solution where $\dot{m} \neq 0$ is given by the sum of the homogeneous solution $(\boldsymbol{b}=0)$ and the non-homogeneous steady state solution

$$
\begin{aligned}
m_{z} & =m_{z, \infty}+\left(m_{0}-p_{z} m_{0}\right) e^{\lambda_{z} t} \\
& =p_{z} m_{0}+\left(m_{0}-p_{z} m_{0}\right) e^{-\frac{t}{T_{1} p_{z}}}
\end{aligned}
$$

where $\lambda_{z}=-1 /\left(\mathrm{T}_{1} p_{z}\right)$ is the third eigenvalue of matrix A. Inserting this equation into Eq. (4) confirms that it is a valid solution. The equation above gives the time-dependent $\mathrm{z}$-magnetization of a spin ensemble after an RF magnetic field is turned on and left on. In deriving it, we have assumed that $\mathrm{T}_{2} \ll \mathrm{T}_{1}$ and that the strength of the RF field is weak such that $\omega_{1} T_{2} \ll 1$. These assumptions give us a concise equation much 
more convenient for saturation experiments in MRFM than the expressions found in the general case. ${ }^{23,24}$

The consequences of Eq. (7) can be seen in Fig. 4. Depending on the precise pulse parameters, even the spins that do not meet the resonance condition by a detuning $\Delta \omega$ can lose (part of) their magnetization due to the RF pulse. The calculation is done assuming $T_{1}=25 \mathrm{~s}$ and $T_{2}=0.15 \mathrm{~ms}$, typical values for copper at $\mathrm{T}=40 \mathrm{mK}$. $^{12}$ The detuning can be translated to a distance to the resonant slice (the region where $\Delta \omega=0$ ) using

$$
d \approx \frac{\Delta \omega}{\gamma \nabla_{r} B_{0}}
$$

where $\nabla_{r} B_{0}$ is the gradient of the magnetic field in the radial direction.

\section{E. Calculation of frequency shifts}

To calculate the frequency shift $\Delta f_{0}$ due to the saturation of the magnetization of the spins in resonance, we first look at the shift of the cantilever resonance frequency due to the coupling with a single spin. For this, we follow a recent theoretical analysis of the magnetic coupling between a paramagnetic spin and the cantilever by De Voogd, Wagenaar, and Oosterkamp. ${ }^{25}$ In our case, where the frequency of the RF pulse $\omega_{R F} \gg \frac{1}{T_{2}}$ and $\omega T_{1} \gg 1$, a single spin induces a stiffness shift given by

$$
\Delta k=\langle m\rangle\left(\left|\mathbf{B}_{\| \mathbf{B}_{0}}^{\prime \prime}\right|+\frac{1}{\mathrm{~B}_{0}}\left|\mathbf{B}_{\perp \mathbf{B}_{0}}^{\prime}\right|^{2}\right)
$$

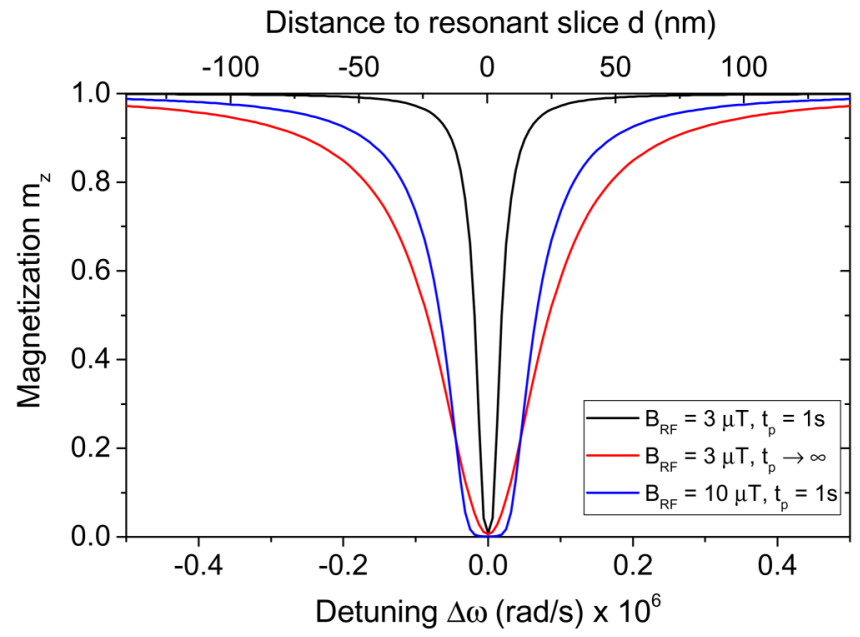

FIG. 4. Calculated magnetization $m_{z}$ after three different RF pulses: in black, after a $1 \mathrm{~s}$ pulse with a strength of $3 \mu \mathrm{T}$, in red, an infinitely long pulse with the same strength, and in blue, a $1 \mathrm{~s}$ pulse with a strength of $10 \mu \mathrm{T}$. The bottom axis shows the detuning $\Delta \omega$, while the top axis shows the corresponding distance to the resonant slice, calculated using Eq. (8) assuming a magnetic field gradient $\nabla_{r} B_{0}=5 \times 10^{4} \mathrm{~T} / \mathrm{m}$.
The primes and double primes refer to the first and second derivatives, respectively, with respect to the fundamental direction of motion of the cantilever. $\left|\mathbf{B}_{\| \mathbf{B}_{0} \mid}^{\prime \prime}\right|$ is the component along $B_{0} .\left|\mathbf{B}_{\perp \mathbf{B}_{0}}^{\prime}\right|$ is the perpendicular component. $\langle m\rangle$ is the mean Boltzmann polarization.

The effect of an RF pulse is to partially remove the magnetization of the spins by an amount given by

$$
\begin{aligned}
\Delta m & =\langle m\rangle-m_{z} \\
& =\langle m\rangle\left(1-p_{z}\right)\left(1-e^{-\frac{t_{p}}{T_{1} p_{z}}}\right),
\end{aligned}
$$

where we set $m_{0}$ equal to $\langle m\rangle$, i.e., we assume the system is in thermal equilibrium before the pulse such that the initial magnetization is equal to the Boltzmann polarization. Please be reminded that $\Delta m$ is position dependent via $p_{z}$ due to the detuning $\Delta \omega$, which increases with the distance to the resonant slice and also depends on the precise RF pulse parameters. We can calculate the total measured frequency shift after an RF pulse by integrating over all spins in the sample including the position dependent demagnetization $\Delta m$

$$
\Delta f_{0}=-\frac{1}{2} \frac{f_{0}}{k_{0}} \rho \int \Delta m\left(\left|\mathbf{B}_{\| \mathbf{B}_{0}}^{\prime \prime}\right|+\frac{1}{\mathrm{~B}_{0}}\left|\mathbf{B}_{\perp \mathbf{B}_{0}}^{\prime}\right|^{2}\right) d \mathrm{~V},
$$

with $\rho=85 \mathrm{spins} / \mathrm{nm}^{3}$ the spin density of copper. Alternatively, one can also sum the contribution of individual voxels, as long as the size of the voxels is small compared to the effective resonant slice width.

\section{FREQUENCY SHIFTS MEASURED IN COPPER}

In this section, we present measured frequency shifts using the higher modes of our cantilever as a source for the RF-field, on one hand to demonstrate that the higher modes can indeed be used to perform full-fledged saturation experiments in MRFM and on the other to give some experimental verifications of the theory presented in Sec. II.

We demonstrate the effectiveness of using the higher modes of the cantilever as an RF field source, by exciting 4 different higher modes of the cantilever by sending a current of $21 \mu \mathrm{A}_{\mathrm{rms}}$ through the RF wire. The frequencies of the selected higher modes are $360 \mathrm{kHz}, 540 \mathrm{kHz}, 756 \mathrm{kHz}$, and $1.009 \mathrm{MHz}$. The position of the resonant slices corresponding to these frequencies is shown in Fig. 5(a). The height of the magnet above the sample determines which of the resonant slices are in the sample and how much signal each of these slices produces. In Fig. 5(b), we show the measured direct frequency shift $\Delta f_{0}$ as a function of the height for each of the higher modes, averaging over 10 single measurements. The error bars are determined by fitting 10 single-shot measurements and calculating the standard deviation of the fitted $\Delta f_{0}$. The solid lines in the figure are the calculated signals based on Eq. (12) using $t_{p}=0.3 \mathrm{~s}$. As the precise amplitude of the mechanically generated RF field is difficult to control since it 

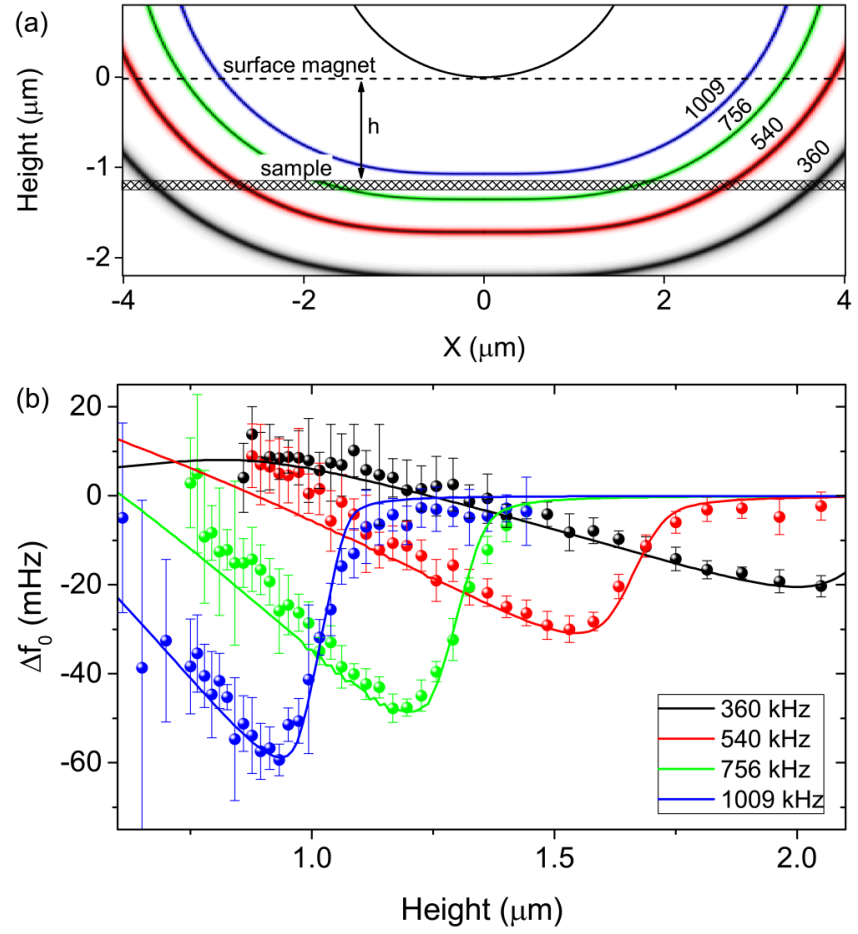

FIG. 5. (a) Positions of the resonant slices corresponding to the higher modes of the cantilever at 360 (black), 540 (red), 756 (green), and 1009 (blue) kHz. The black circle at the top of the image represents the cantilever magnet (radius $1.7 \mu \mathrm{m}$, to scale). (b) Direct frequency shift $\Delta f_{0}$ versus height $h$ after exciting the spins by using the RF wire to drive the higher modes of the cantilever indicated in (a), measured at $T=30 \mathrm{mK}$. Solid lines are the calculated signals for a pulse duration $t_{p}=0.3 \mathrm{~s}$, and $B_{R F}$ a free parameter. The error bars indicate the standard deviation of 10 single-shot measurements.

depends on the distance between the magnet and the RF wire, the height of the magnet above the sample, and the Q-factor of the higher mode, the strength of the RF field is the only free fitting parameter. From the fits, we obtain fields of $38,35,38$, and $33 \mu \mathrm{T}$ for the 4 higher modes as mentioned before. Evidently, the different higher modes enter the sample at the predicted heights, with the correct overall magnitude of the direct frequency shift. The small deviation between the data and calculation at the lower heights probably results from a slightly changing $B_{R F}$. This measurement can be considered as a crude one-dimensional scan of the sample. Furthermore, considering that the current of $21 \mu \mathrm{A}_{\mathrm{rms}}$ corresponds to a field of only $0.2 \mu \mathrm{T}$ at the position of the cantilever, $7 \mu \mathrm{m}$ away from the RF wire, this measurement indicates that using the higher modes to generate the RF field results in an amplification of the RF field strength of more than a factor of 160. No heating was observed on the sample holder, indicating a dissipated power of $<1 \mathrm{nW}$.

We can further demonstrate the effect of the pulse parameters on the effective resonant slice width by doing a variation on the previous experiment. We now keep the sample at a constant height and vary the duration of the RF current used to excite each of the higher modes in order to broaden the resonant slice. By comparing the measured increase of the signal for the various higher modes to the signal we expect from Eqs. (7) and (12), we can confirm the applicability of these equations. This experiment is shown in Fig. 6. The inset shows the calculated frequency shift as a function of the RF frequency, as well as the position of the higher modes. From the inset we see that for short pulses (a narrow resonant slice), we expect no signal from the $540 \mathrm{kHz}$ and $1.299 \mathrm{MHz}$ higher modes, some signal from the $756 \mathrm{kHz}$ higher mode, and most signal from the $1.009 \mathrm{MHz}$ higher mode. This behaviour is also observed in the main figure, where the solid lines are the calculated frequency shifts based on Eq. (12). As $t_{p}$ is increased, even the resonant slices whose center is not in the sample broaden enough that off-resonant spins start to create measurable frequency shifts, with a good correlation between theory and experiment. The mismatch between the measured and calculated signal for very short pulse durations is attributed to the large Q-factor of the higher modes, which can be as high as $10^{6}$, resulting in characteristic time constants of up to $1 \mathrm{~s}$. In that case, driving the higher mode for a very short time still results in a long effective pulse duration determined by the slow ringdown of the higher mode.

\section{DEMONSTRATION OF VOLUME SENSITIVITY}

As shown in Fig. 2, we have a very clean frequency noise spectrum. To make full use of this, we have attempted to determine our optimal frequency resolution. To achieve this,

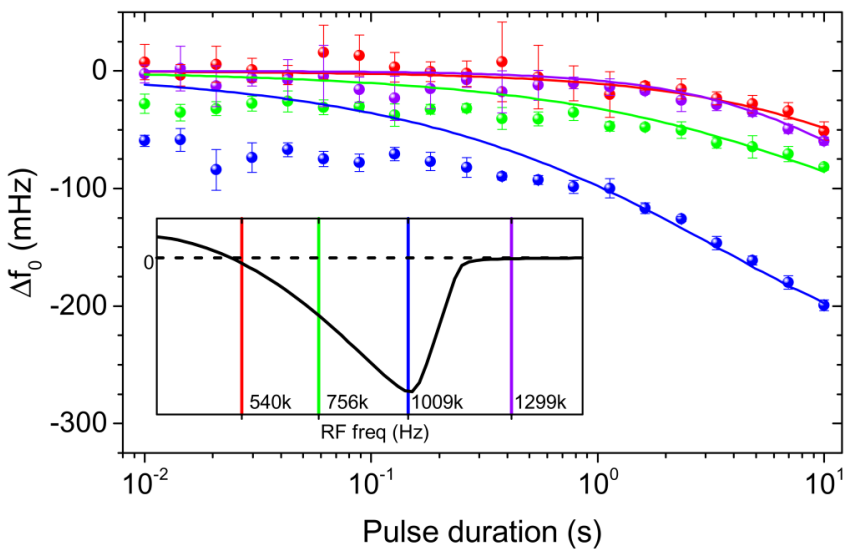

FIG. 6. Effect of the excitation pulse duration $t_{p}$ on the measured direct frequency shift $\Delta f_{0}$ for the higher modes of the cantilever at 540 (red), 756 (green), 1009 (blue), and 1299 (purple) kHz, measured at $h=0.95 \mu \mathrm{m}$ and $T=30 \mathrm{mK}$. The inset shows the calculated direct frequency shift as a function of the RF frequency and also shows the position of the higher modes in this calculation. As $t_{p}$ increases, the resonant slice broadens and the direct frequency shift increases as expected from the resonant slice positions indicated in the inset. The error bars indicate the standard deviation of 5 single-shot measurements. 
we make a small adjustment to the measurement scheme, by switching off the cantilever drive a couple of seconds before we apply the RF pulse. The amplitude of the fundamental mode decays quickly due to the relatively low Q-factor of the fundamental mode close to the sample. By the time the pulse is sent, the amplitude of the cantilever is thermally limited to less than $0.1 \mathrm{~nm}$. Directly after the pulse, the cantilever drive is switched back on to measure the resonance frequency shift. In this way, we prevent broadening of the resonant slice due to the cantilever amplitude of about $30 \mathrm{~nm}_{\text {rms }}$ and are able to achieve very narrow resonant slices. Figure 7 shows the relaxation curve measured at $\mathrm{T}=21 \mathrm{mK}$ and $h=1.0 \mu \mathrm{m}$, after an $882 \mathrm{kHz}$ RF pulse with $\mathrm{B}_{\mathrm{RF}}=172 \mu \mathrm{T}$ and $t_{p}=80 \mu \mathrm{s}$. The blue curve shows the result of 410 averages with a total measurement time of over $10 \mathrm{~h}$, while the red curve is a fit to the data following Eq. (2), from which we extract a direct frequency shift of $-5.4 \mathrm{mHz}$. The inset shows the difference between the measured data and the fit, indicating that we can measure the frequency shift with a standard deviation of 0.1 $\mathrm{mHz}$, consistent with the integrated frequency noise calculated from Fig. 2 and the number of averages.

We can try to estimate the total detection volume that was necessary to generate this signal. In order to do so, we make the simplifying assumption that there exists a critical detuning $\Delta \omega_{\mathrm{C}}$ such that all spins at a detuning smaller than the critical detuning (i.e., spins that feel a magnetic field between $B_{0}-\Delta \omega_{C} / \gamma$ and $\left.B_{0}+\Delta \omega_{C} / \gamma\right)$ are fully saturated, and spins at a detuning larger than the critical detuning are completely unaffected by the pulse. We then calculate the signal for various values of $\Delta \omega_{C}$ until we find the value for which the calculation matches the experiment. By dividing the sample in small voxels and summing all voxels that satisfy

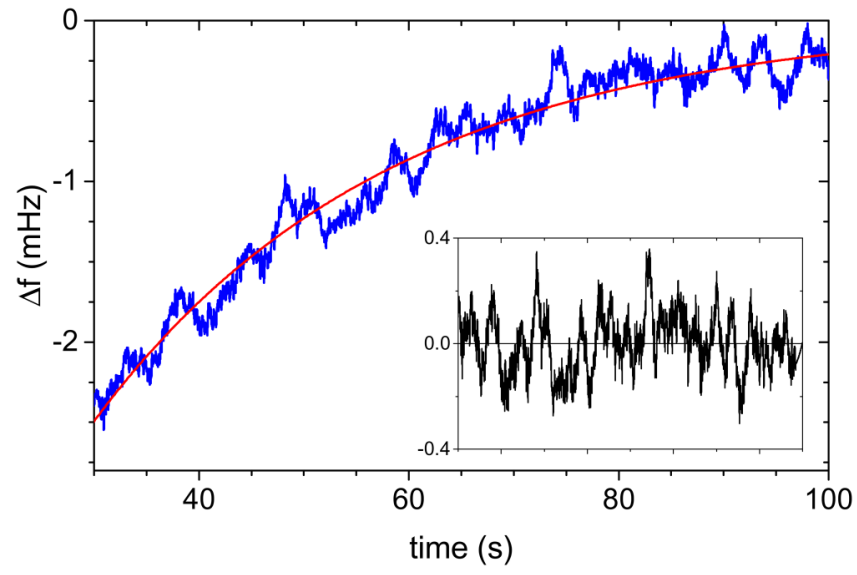

FIG. 7. Relaxation curve (1 $\mathrm{Hz}$ low-pass filter, 410 averages) measured at $h=1.0 \mu \mathrm{m}$ and $T=21 \mathrm{mK}$, for a pulse at frequency $882 \mathrm{kHz}$ with $B_{R F}=172 \mu \mathrm{T}$ and $t_{p}=80 \mu \mathrm{s}$. The solid red line is a fit to Eq. (2), from which we extract $\Delta f_{0}=-5.4 \mathrm{mHz}$. The inset shows the difference between the data and the exponential fit, indicating a standard deviation of the measured frequency shift of $0.1 \mathrm{mHz}$. the condition specified above for the correct $\Delta \omega_{C}$, we find an estimate for the detection volume.

For the data presented in Fig. 7, we find that this signal is the result of a critical detuning $\Delta \omega_{\mathrm{C}} /(2 \pi)=2.1 \mathrm{kHz}$, equivalent to a resonant slice with a full width of approximately $4 \mathrm{~nm}$. This corresponds to a total detection volume of $(152 \mathrm{~nm})^{3}$, with a noise floor equal to $(40 \mathrm{~nm})^{3}$. This volume contains a total of $5.5 \times 10^{6}$ spins at a Boltzmann polarization of about $0.3 \%$, corresponding to about $1.6 \times 10^{4}$ fully polarized copper nuclear spins.

Note that for very small resonant slice widths, spin diffusion might be a relevant factor. ${ }^{26}$ However, only spin diffusion during the RF pulse influences the size of the detection volume. Since in this experiment the pulse duration is only $80 \mu \mathrm{s}$, we calculate that the spin diffusion length is less than $0.1 \mathrm{~nm}$ (see the supplementary material for details about the expected spin diffusion), much smaller than the estimated resonant slice width of $4 \mathrm{~nm}$.

\section{IMAGING PROTONS}

With the volume sensitivities achieved on copper as demonstrated in Sec. IV, it is worthwhile to discuss what such an experiment would look like for a sample containing protons, the prime target spin for imaging purposes. Therefore, in this section, we will calculate the signals that can be expected from a proton-rich sample, under the assumption that it is possible to achieve the same low frequency noise as in the current experiment on copper. ${ }^{1} \mathrm{H}$ spins have spin $\mathrm{S}=1 / 2$, a gyromagnetic ratio $\gamma_{\mathrm{H}} /(2 \pi)=42.6 \mathrm{MHz} / \mathrm{T}$, and a magnetic moment $\mu_{\mathrm{H}}=1.41 \times 10^{-26} \mathrm{~J} / \mathrm{T}$. For MRFM, proton spins are generally a bit more favourable than copper spins, as the higher gyromagnetic ratio and magnetic moment mean a higher Boltzmann polarization and a larger coupling between a single spin and the cantilever. We assume a proton spin density $\rho_{\mathrm{H}}=50 \mathrm{spins} / \mathrm{nm}^{3}$, a typical value for biological tissue and polymers. ${ }^{5,19}$ Furthermore, we assume $T_{1}=30 \mathrm{~s}$ and $T_{2}=0.1 \mathrm{~ms}$. Note that the exact values for the relaxation times do not matter that much as long as the conditions used for the derivation of Eqs. (7) and (9) are met, and the RF pulse duration is short compared to $\mathrm{T}_{1}$.

We calculate the total volume necessary to get a frequency shift of $1.8 \mathrm{mHz}\left(\mathrm{V}_{\mathrm{ss}}\right)$, a signal that can be measured in a single shot experiment assuming the SNR achieved on copper, and $0.5 \mathrm{mHz}$, which can be measured within $30 \mathrm{~min}$ ( $\sim 15$ averages). The results can be found in Table I. We

TABLE I. Calculated volume sensitivities $V_{s s}$ (volume required for a $1.8 \mathrm{mHz}$ frequency shift) and $V_{30 \mathrm{~min}, D N P}$ (volume required for a $0.5 \mathrm{mHz}$ frequency shift). Calculations are done for sample temperature $T=21 \mathrm{mK}$ and RF frequency $\omega_{R F} /(2 \pi)=3.5 \mathrm{MHz}$. The radial magnetic field gradient $\nabla_{r} B_{0}$ is calculated at 50 $\mathrm{nm}$ below the surface of the sample.

\begin{tabular}{lcccc}
\hline \hline $\boldsymbol{R}_{\mathbf{0}}(\boldsymbol{\mu} \mathrm{m})$ & $\boldsymbol{h}(\boldsymbol{\mu \mathrm { m } )}$ & $\nabla_{\boldsymbol{r}} \boldsymbol{B}_{\mathbf{0}}(\boldsymbol{\mu T} \mathrm{T} / \mathrm{nm})$ & $\boldsymbol{V}_{\boldsymbol{s s}}$ & $\boldsymbol{V}_{\mathbf{3 0 \mathrm { min }}}$ \\
\hline 1.7 & 1.00 & 100 & $(84 \mathrm{~nm})^{3}$ & $(55 \mathrm{~nm})^{3}$ \\
1.0 & 0.56 & 170 & $(59 \mathrm{~nm})^{3}$ & $(39 \mathrm{~nm})^{3}$ \\
0.5 & 0.24 & 370 & $(39 \mathrm{~nm})^{3}$ & $(25 \mathrm{~nm})^{3}$ \\
\hline \hline
\end{tabular}


considered three different experimental configurations, where we vary the size of the magnet in order to increase the field gradients and thereby the signal per spin. The first configuration is a replication of the experimental parameters as used for the copper measurement from Fig. 7: A saturation experiment performed at a height of $1.0 \mu \mathrm{m}$ and a temperature of $21 \mathrm{mK}$. The optimal signal at this height is found for an RF frequency of $3.5 \mathrm{MHz}$ (about a factor of 4 higher than the RF frequency used for the copper due to the higher gyromagnetic ratio). The other two configurations are simulations with magnets with radii $1.0 \mu \mathrm{m}$ and $0.5 \mu \mathrm{m}$. To make a fair comparison, we calculate the signal for the same Larmor frequency $3.5 \mathrm{MHz}$, which dictates measurement heights of $0.56 \mu \mathrm{m}$ and $0.24 \mu \mathrm{m}$. All unmentioned parameters are kept constant. The predicted detection volumes for the different configurations are shown in Table I.

Clearly, decreasing the size of the magnetic particle will enhance the volume sensitivity but there is a fundamental limit: the experiment described here relies on removing the Boltzmann polarization of the sample, but as the detection volume goes down, we enter the regime where the statistical polarization becomes dominant. The critical volume $V_{c}$ for this transition is given by ${ }^{27}$

$$
\mathrm{V}_{\mathrm{c}}=\frac{4}{\rho_{\mathrm{H}}}\left(\frac{k_{\mathrm{B}} \mathrm{T}}{\hbar \gamma \mathrm{B}_{0}}\right)^{2},
$$

where it is assumed that the thermal energy is much larger than the Zeeman splitting. For a temperature of $21 \mathrm{mK}$ and a Larmor frequency of $3.5 \mathrm{MHz}, \mathrm{V}_{\mathrm{c}} \sim(11 \mathrm{~nm})^{3}$. Below this detection volume, measurements of the direct frequency shift would average to zero.

However, further enhancement of the volume sensitivity can still be achieved by increasing the Boltzmann polarization of the protons. This can be done by working at higher Larmor frequencies by decreasing the tip-sample separation or by applying a strong external magnetic field. An external magnetic field of $8 \mathrm{~T}$ would increase the Boltzmann polarization by roughly a factor of 100 , but applying external magnetic fields in combination with our SQUID-based detection is challenging due to our extreme sensitivity to magnetic noise. An appealing alternative is to use dynamical nuclear polarization (DNP), as was recently demonstrated for MRFM by Issac et al. For suitable samples, e.g., nitroxide-doped polystyrene, DNP can be used to transfer polarization from electron spins to nuclei. The maximum enhancement of the nuclear polarization that can be achieved using this mechanism is given by $\epsilon=\gamma_{e} / \gamma_{\mathrm{H}}=660$. However, for protons at a Larmor frequency of $3.5 \mathrm{MHz}$ and temperature of $21 \mathrm{mK}$, the initial Boltzmann polarization is about $0.4 \%$, so our maximal enhancement is limited to a factor of 250. Table II shows the calculated volume sensitivities if we are able to use DNP to enhance the nuclear polarization for the cases where we achieve DNP efficiencies of $10 \%$ and $100 \%$. Even for the more realistic assumption of $10 \%$ efficiency, we find that a volume sensitivity below $(10 \mathrm{~nm})^{3}$ could be possible. This voxel size would
TABLE II. Calculated volume sensitivities $V_{s s, D N P}$ and $V_{30 \text { min,DNP }}$ including DNP to enhance the nuclear polarization with an efficiency $D N P_{\text {eff. }}$ Calculations are done for sample temperature $T=21 \mathrm{mK}$ and RF frequency $\omega_{R F} /(2 \pi)=3.5 \mathrm{MHz}$.

\begin{tabular}{lcccc}
\hline \hline $\boldsymbol{R}_{\mathbf{0}}(\boldsymbol{\mu} \mathrm{m})$ & $\boldsymbol{h}(\boldsymbol{\mu} \mathrm{m})$ & $\boldsymbol{D N P}_{\text {eff }}(\%)$ & $\boldsymbol{V}_{\boldsymbol{s s}, \text { DNP }}$ & $\boldsymbol{V}_{30 \mathrm{~min}, \text { DNP }}$ \\
\hline 1.7 & 1.00 & 10 & $(21 \mathrm{~nm})^{3}$ & $(14 \mathrm{~nm})^{3}$ \\
1.7 & 1.00 & 100 & $(13 \mathrm{~nm})^{3}$ & $(8.7 \mathrm{~nm})^{3}$ \\
1.0 & 0.56 & 10 & $(15 \mathrm{~nm})^{3}$ & $(10 \mathrm{~nm})^{3}$ \\
1.0 & 0.56 & 100 & $(9.4 \mathrm{~nm})^{3}$ & $(6.1 \mathrm{~nm})^{3}$ \\
0.5 & 0.24 & 10 & $(9.6 \mathrm{~nm})^{3}$ & $(6.2 \mathrm{~nm})^{3}$ \\
0.5 & 0.24 & 100 & $(6.1 \mathrm{~nm})^{3}$ & $(4.0 \mathrm{~nm})^{3}$ \\
\hline \hline
\end{tabular}

make imaging based on measurements of the Boltzmann polarization a viable approach to image biological samples, without the demand for high RF field amplitudes and continuous application of this field, as was the case for previous amplitude-based imaging. ${ }^{5}$

Of course, there are some potential pitfalls that should be considered. First of all, we have assumed that the frequency noise spectrum shown in Fig. 2 can be maintained. However, large $1 / f$ noise has been reported at $4 \mathrm{~K}$ on insulating samples like polymers, attributed to dielectric fluctuations. ${ }^{28,29}$ This frequency noise scales with the square of the charge difference between the sample and the tip. Therefore, we believe that it can be avoided, either by properly grounding both the tip and the sample, but also by biasing the tip to tune away any charge difference. ${ }^{30,31}$

A second limitation is that for the current experiment, we require $T_{1}$ times to be between several seconds and minutes. When $T_{1}$ is shorter than several seconds, it becomes comparable to other time constants in our setup (e.g., the thermal time constant of the sample holder), making it difficult to extract the signal. When $\mathrm{T}_{1}$ becomes longer than minutes, averaging measurements to increase the SNR will become very time-consuming, although the total measurement time may come down by using multiple resonant slices. ${ }^{32,33}$ Plus, as the duration of a measurement increases, $1 / f$ noise will increasingly become a limiting factor. $T_{1}$ times within the desired range for suitable proton samples are reported at low temperatures. ${ }^{19,34}$ For very pure samples with long $\mathrm{T}_{1}$ times, appropriate doping of the sample with impurities can be used to reduce the relaxation time. ${ }^{35}$

The final challenge is to maintain the low operating temperatures required for the low frequency noise floor while sending RF pulses in the MHz range. The power dissipated by the RF pulse, even when using a superconducting RF wire, increases with the frequency. To apply a $0.1 \mathrm{mT}$ RF pulse at a sample located $5 \mu \mathrm{m}$ from the RF wire at $3.5 \mathrm{MHz}$, we measure a dissipation of approximately $3 \mu \mathrm{W}$ in our setup. A continuous power pulse with this level of dissipation would locally heat the sample to over $100 \mathrm{mK}$. We can avoid this source of dissipation by using the higher modes of the cantilever, which can be excited up to the 15 th mode at $4.4 \mathrm{MHz}$ and possibly beyond. In Fig. 8, we show the frequencies of the higher flexural modes together with the calculated frequencies obtained from finite element calculations. The estimated dissipation 


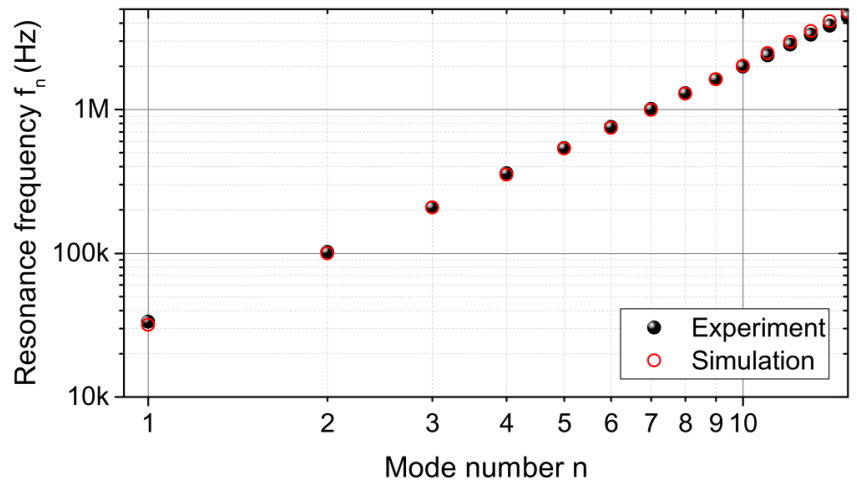

FIG. 8. Measured higher mode resonance frequencies of the cantilever, together with the mode frequencies obtained from finite element calculations. The highest resonance mode investigated is the 15th mode located at $f_{15}=4.4 \mathrm{MHz}$. In the simulations, we only consider higher modes that vibrate in the soft direction of the cantilever.

from the motion of a higher mode is well below $1 \mathrm{fW}$, since we measure the higher modes to have Q-factors approaching one million. Note that exciting the higher modes becomes harder for higher mode numbers, as the rotation angle of the magnet (that partially determines the magnitude of the generated RF field) scales with the inverse of the torsional stiffness $\kappa_{n} \propto n^{4}$. For the presented mode numbers, this can be compensated by increasing the amplitude of the driving force. We do expect, however, that non-linearities of the cantilever will be the fundamental limit for the maximum fields that we can generate using the higher modes. ${ }^{13}$

\section{CONCLUSIONS}

We have used the time-dependent solution of the Bloch equation to derive a concise equation to calculate the frequency shifts in MRFM experiments and applied this to saturation experiments on a thin copper film. By using the higher modes of the cantilever as a source for the RF fields, we have demonstrated that it is possible to make one-dimensional scans of the copper film with near-negligible dissipation, and that the measured direct frequency shifts are well reproduced by the presented theory. Finally, we have shown that we have measured a frequency-shift signal with a volume sensitivity of $(40 \mathrm{~nm})^{3}$. We have done all this at temperatures as low as 21 $\mathrm{mK}$, made possible by the SQUID-based detection of the cantilever motion and the low power saturation protocol in combination with the mechanical generation of the RF fields.

The achieved volume sensitivity opens up the way for imaging based on measurements of the Boltzmann polarization, which could allow for high resolution imaging due to the direct gain from lower temperatures, and the favourable averaging compared to statistical polarization based imaging. We have shown that modest technical changes to our current setup can allow for experiments on protons with a spatial resolution of $(25 \mathrm{~nm})^{3}$ and that increasing the polarization, for instance, using DNP can improve the resolution even further to below $(10 \mathrm{~nm})^{3}$. The magnet-on-tip geometry allows for a larger choice in available samples, as it is still an open question whether interesting biological samples can be attached to an ultrasoft MRFM cantilever for approaches using the sample-on-tip geometry. When it is possible to measure on different samples with the same low frequency noise as achieved in the current experiment, high-resolution Boltzmann-polarization-based magnetic resonance imaging at millikelvin temperatures in a magnet-on-tip geometry could become a reality.

\section{SUPPLEMENTARY MATERIAL}

See the supplementary material for the relevant NMR properties of copper and details about spin diffusion in copper.

\section{ACKNOWLEDGMENTS}

The authors thank K. Heeck, M. Camp, G. Koning, F. Schenkel, D. J. van der Zalm, J. P. Koning, and L. Crama for technical support. The authors thank D. J. Thoen, T. M. Klapwijk, and A. Endo for providing us with the NbTiN. The authors thank T. H. A. van der Reep for valuable discussions and proofreading the manuscript. This work is supported by the Netherlands Organisation for Scientific Research (NWO) through a VICI fellowship to T.H.O. and through the Nanofront program.

\section{REFERENCES}

${ }^{1}$ O. Züger and D. Rugar, Appl. Phys. Lett. 63, 2496 (1993).

${ }^{2}$ D. Rugar, O. Züger, S. Hoen, C. S. Yannoni, H. Vieth, and R. D. Kendrick, Science 264, 1560 (1994).

${ }^{3}$ J. A. Sidles, J. L. Garbini, K. J. Bruland, D. Rugar, O. Züger, S. Hoen, and C. S. Yannoni, Rev. Mod. Phys. 67, 249 (1995).

${ }^{4}$ D. Rugar, R. Budakian, H. J. Mamin, and B. W. Chui, Nature 430, 329 (2004).

${ }^{5}$ C. L. Degen, M. Poggio, H. J. Mamin, C. T. Rettner, and D. Rugar, Proc. Natl. Acad. Sci. U.S.A. 106, 1313 (2009).

${ }^{6}$ W. Rose, H. Haas, A. Q. Chen, N. Jeon, L. J. Lauhon, D. G. Cory, and R. Budakian, Phys. Rev. X 8, 011030 (2018).

${ }^{7}$ M. Poggio, C. L. Degen, C. T. Rettner, H. J. Mamin, and D. Rugar, Appl. Phys. Lett. 90, 263111 (2007).

${ }^{8}$ J. M. Nichol, E. R. Hemesath, L. J. Lauhon, and R. Budakian, Phys. Rev. B 85, 054414 (2012).

${ }^{9}$ S. R. Garner, S. Kuehn, J. M. Dawlaty, N. E. Jenkins, and J. A. Marohn, Appl. Phys. Lett. 84, 5091 (2004).

${ }^{10}$ H. J. Mamin, M. Poggio, C. L. Degen, and D. Rugar, Nat. Nanotechnol. 2, 301 (2007).

${ }^{11}$ D. A. Alexson, S. A. Hickman, J. A. Marohn, and D. D. Smith, Appl. Phys. Lett. 101, 022103 (2012).

12J. J. T. Wagenaar, A. M. J. den Haan, J. M. de Voogd, L. Bossoni, T. A. de Jong, M. de Wit, K. M. Bastiaans, D. J. Thoen, A. Endo, T. M. Klapwijk, J. Zaanen, and T. H. Oosterkamp, Phys. Rev. Appl. 6, 014007 (2016).

${ }^{13}$ J. J. T. Wagenaar, A. M. J. den Haan, R. J. Donkersloot, F. Marsman, M. de Wit, L. Bossoni, and T. H. Oosterkamp, Phys. Rev. Appl. 7, 024019 (2018).

${ }^{14}$ B. W. Chui, Y. Hishinuma, R. Budakian, H. J. Mamin, T. W. Kenny, and D. Rugar, in TRANSDUCERS, the 12th International Conference on Solid-State Sensors, Actuators and Microsystems (IEEE, 2003), Vol. 2, pp. 1120-1123.

${ }^{15}$ O. Usenko, A. Vinante, G. H. C. J. Wijts, and T. H. Oosterkamp, Appl. Phys. Lett. 98, 133105 (2011). 
${ }^{16}$ D. J. Thoen, B. G. C. Bos, E. A. F. Haalebos, T. M. Klapwijk, J. J. A. Baselmans, and A. Endo, IEEE Trans. Appl. Supercond. 27(4), 1 (2017).

${ }^{17}$ A. M. J. Den Haan, G. H. C. J. Wijts, F. Galli, O. Usenko, G. J. C. Van Baarle, D. J. Van Der Zalm, and T. H. Oosterkamp, Rev. Sci. Instrum. 85, 035112 (2014).

${ }^{18}$ E. S. Meyer, I. F. Silvera, and B. L. Brandt, Rev. Sci. Instrum. 60, 2964 (1989).

${ }^{19}$ C. E. Isaac, C. M. Gleave, P. T. Nasr, H. L. Nguyen, E. A. Curley, J. L. Yoder, E. W. Moore, L. Chen, and J. A. Marohn, Phys. Chem. Chem. Phys. 18, 8806 (2016).

${ }^{\mathbf{2 0}} \mathrm{A}$. Abragam, Principles of Nuclear Magnetism, International Series of Monographs on Physics (Clarendon Press, Oxford, 1961), p. 128.

${ }^{21}$ F. Bloch, Phys. Rev. 70, 460 (1946).

${ }^{22}$ C. P. Slichter, Principles of Magnetic Resonance, Springer Series in Solid-State Sciences Vol. 1 (Springer, Berlin, Heidelberg, 1990).

${ }^{23}$ R. V. Mulkern and M. L. Williams, Med. Phys. 20, 5 (1993).

${ }^{24} \mathrm{~K}$. Murase and N. Tanki, Magn. Reson. Imaging 29, 126 (2011).

${ }^{25} \mathrm{~J}$. M. De Voogd, J. J. T. Wagenaar, and T. H. Oosterkamp, Sci. Rep. 7, 42239 (2017).

26. Cardellino, N. Scozzaro, M. Herman, A. J. Berger, C. Zhang, K. C. Fong, C. Jayaprakash, D. V. Pelekhov, and P. C. Hammel, Nat. Nanotechnol. 9, 343 (2014).
${ }^{27}$ B. E. Herzog, D. Cadeddu, F. Xue, P. Peddibhotla, and M. Poggio, Appl. Phys. Lett. 105, 043112 (2014).

${ }^{28}$ S. M. Yazdanian, J. A. Marohn, and R. F. Loring, J. Chem. Phys. 128, 224706 (2008).

${ }^{29}$ N. Hoepker, S. Lekkala, R. F. Loring, and J. A. Marohn, J. Phys. Chem. B 115, 14493 (2011).

${ }^{30}$ S. M. Yazdanian, N. Hoepker, S. Kuehn, R. F. Loring, and J. A. Marohn, Nano Lett. 9, 2273 (2009).

${ }^{31}$ E. W. Moore, S. Lee, S. A. Hickman, S. J. Wright, L. E. Harrell, P. P. Borbat, J. H. Freed, and J. A. Marohn, Proc. Natl. Acad. Sci. U.S.A. 106, 22251 (2009).

${ }^{32}$ T. H. Oosterkamp, M. Poggio, C. L. Degen, H. J. Mamin, and D. Rugar, Appl. Phys. Lett. 96, 083107 (2010).

${ }^{33}$ B. A. Moores, A. Eichler, Y. Tao, H. Takahashi, P. Navaretti, and C. L. Degen, Appl. Phys. Lett. 106, 213101 (2015).

${ }^{34}$ L. Chen, J. G. Longenecker, E. W. Moore, and J. A. Marohn, Appl. Phys. Lett. 102, 132404 (2013).

${ }^{35} \mathrm{H}$. H. Grapengeter, R. Kosfeld, and H. W. Offergeld, Polymer 21, 829 (1980).

${ }^{36} \mathrm{M}$. de Wit, G. Welker, K. Heeck, F. M. Buters, H. J. Eerkens, G. Koning, H. van der Meer, D. Bouwmeester, and T. H. Oosterkamp, Rev. Sci. Instrum. 90(1), 015112 (2019). 